\title{
Efeito da intervenção terapêutica ocupacional junto a crianças com déficts de percepção visual, cordenação motora e integração visuo-motora
}

Rita de CássiaTibério Araújo* Mariana Dutra Zafani** Débora Morais Pereira***

\section{Resumo}

Para que o aluno com necessidades educacionais especiais participe ativamente da escola são necessários investimentos efetivos e sistemáticos, envolvendo a comunidade escolar como um todo. O terapeuta ocupacional é um dos profissionais que pode favorecer o processo de inclusão destes alunos. Este estudo teve como objetivo discutir o efeito da intervenção terapêutica ocupacional junto a duas crianças com deficiência incluídas no ensino regular, com déficits de percepção visual, coordenação motora e integração visuo-motora. Para a avaliação das habilidades de percepção visual, coordenação motora e integração visuo-motora foi utilizado o Beery-Buktenica Developmental Test of Visual-Motor Integration. Em virtude dos déficits apresentados nas funções investigadas foi verificada a necessidade de aplicação de um programa de intervenção terapêutico ocupacional visando à melhora dessas funções. Após aplicação do programa, o teste foi reaplicado. Os resultados apontaram para a melhora de todas as funções consideradas deficitárias, sinalizando a importância do treinamento de desempenho.

Palavras-chave: Terapia ocupacional; Educação especial; Integração visuomotora.

Occupational therapy intervention effect for children with deficits in visual perception, motor coordination and visualmotor integration

\begin{abstract}
To students with special educational needs participate actively at school are required effective and systematic investment, involving the school community as a whole. The occupational therapist is one of the professionals who can facilitate this student inclusion process. This study aimed to discuss the occupational

\footnotetext{
* Professora Doutora do Curso de Terapia Ocupacional da Faculdade de Filosofia e Ciências, UNESP, campus de Marília. Marília, São Paulo.

** Mestranda no Programa de Pós-Graduação em Educação da Faculdade de Filosofia e Ciências, UNESP, campus Marília. Marília, São Paulo.

*** Mestranda no Programa de Pós-Graduação em Educação da Faculdade de Filosofia e Ciências, UNESP, campus Marília, Marília, São Paulo.
} 
therapy intervention effects with two disability children with deficits in visual perceptual skills, motor coordination and visual motor integration, that was included in regular education. The Beery-Buktenica Developmental Test of Visual Motor Integration was use to evaluate visual perceptual skills, motor coordination and visual-motor integration. Because the deficits presented in the functions investigates was identified the need of an occupational therapy intervention program designed to improve the performance in theses functions. After the program, the test was reapplied. The results pointed to an improvement of all functions considered deficient. These results highlight to the training importance to improve the performance in abilities evaluated.

Keywords: Occupational therapy; Special education; Visual-motor integration.

\section{Introdução}

A possibilidade de acesso à escolarização traz ganhos inestimáveis à pessoa com deficiência. Entre 1998 e 2006 houve um crescimento das matrículas dessa população em classes comuns de escolas de ensino regular. Segundo dados da Secretaria de Educação Especial, "estão em classes comuns 375.772 estudantes com deficiência, transtornos globais do desenvolvimento e altas habilidades ou superdotação" (BRASIL, 2012, s/p.). Estes números indicam que pessoas com deficiência estão cada vez mais incluídas nas escolas regulares, no entanto, colocar o aluno dentro da escola não é suficiente (AMIRALIAN; JURDI, 2006).

A matrícula de alunos com deficiência na escola regular de ensino não é suficiente para garantir o aprendizado e socialização desses alunos. A inclusão é um processo que envolve não apenas o acesso, mas em especial, a permanência e o sucesso na escola (MENDES, 2002). Para que a escola não seja um espaço de exclusão ou de inclusão perversa são necessários apoios comuns a todos os alunos (ROCHA; LUIZ; ZULIAN, 2003).

De acordo com Martins (2001) para que a escola se torne inclusiva, é preciso que ela, além de dar abertura para a matrícula de forma não restritiva, incentive a participação ativa e a aprendizagem de todos, sendo necessário para isso um investimento efetivo e sistemático, envolvendo a comunidade escolar como um todo: técnicos, funcionários, professores, alunos, pais e, até mesmo, uma mudança expressiva na sua própria proposta educacional. Considerando que a inclusão é um processo dinâmico também são necessários para sua efetivação constantes avaliações, reflexões e discussões com as práticas e vivências (COSTABILE; BRUNELLO, 2005).

Segundo o artigo $6^{\circ}$, parágrafo III da Resolução CNE/CEB № 2, de 11 de setembro de 2001 que institui Diretrizes Nacionais para a Educação Especial na Educação Básica para o assessoramento técnico e avaliação do aluno no 
processo de ensino e aprendizagem, a escola deve contar com a cooperação dos serviços de saúde (BRASIL, 2001).

O terapeuta ocupacional é um dos profissionais da saúde que pode contribuir para facilitar a adaptação e a participação social de crianças com necessidades educacionais no ambiente escolar. Segundo Law (2002) o terapeuta ocupacional tem seu foco principal na habilidade do indivíduo para realizar tarefas essenciais a fim de permitir sua participação em diferentes contextos. Para a autora, no caso do ambiente escolar essas habilidades estão relacionadas a tarefas como organizar o material escolar, escrever, colorir, recortar e até mesmo manejar utensílios e materiais necessários para se alimentar independentemente.

Sob parâmetros de funcionalidade, para a realização das atividades escolares algumas habilidades psicomotoras precisam ser consideradas. Segundo Nogueira, Carvalho e Pessanha (2007) defasagens no desenvolvimento das habilidades psicomotoras têm consequências no desempenho e na construção de leitura e escrita, principalmente nas séries iniciais do ensino fundamental. Entre as habilidades psicomotoras está a habilidade de integração visuo-motora que se refere à interação entre o ato motor (coordenação motora) e a estimulação visual percebida (percepção visual). Esta habilidade depende da participação de diferentes centros nervosos motores e sensoriais que se traduzem pela organização de programas motores e pela intervenção de diversas sensações oriundas dos receptores sensoriais, articulares e cutâneos do membro requerido (ROSA NETO, 2002).

Segundo Amaral e Barbosa (2009) a habilidade visuo-motora é um aspecto da psicomotricidade que prepara a criança para a aprendizagem da escrita e quando não trabalhada pode acarretar dificuldades como inabilidade da letra cursiva, letra ilegível e mau uso do lápis.

$\mathrm{Na}$ literatura nacional e estrangeira, são encontrados estudos de terapeutas ocupacionais que destacam a importância da integração visuo-motora, percepção visual e coordenação motora na aprendizagem das habilidades acadêmicas (MAGALHÃES et al., 2009; DALY, KELLEY, KRAUSS, 2003; CORNHILL; CASE-SMITH, 1996; WEIL; AMUNDSON, 1994).

Portanto, há indícios de que esses três componentes de desempenho são considerados fundamentais na execução das atividades escolares, e consequentemente estão estreitamente relacionados-com as habilidades de escrita, leitura, matemática, entre outras (ZAFANI, ARAÚJO, 2010).

Considerando a importância da percepção visual, coordenação motora e integração visuo-motora para o sucesso nas atividades acadêmicas e a ocorrência recorrente de falhas nessa habilidade manifestadas por crianças com algum tipo de deficiência, o presente trabalho teve como objetivo discutir o efei- 
Rita de CássiaTibério Araújo - Mariana Dutra Zafani

Débora Morais Pereira

to da intervenção terapêutica ocupacional junto a crianças com déficits de percepção visual, coordenação motora e integração visuo-motora.

\section{Método}

Participantes e contexto de coleta de dados

Participaram do estudo duas crianças com oito anos de idade, com diagnósticos de paralisia cerebral atáxica (P1) e Síndrome de Russel-Silver (P2), recrutadas nos serviços de terapia ocupacional de uma clínica vinculada a uma universidade pública, do interior do Estado de São Paulo.

\section{Instrumentos}

Para avaliação das habilidades de percepção visual, coordenação motora e integração visuo-motora foi utilizado o Beery-Buktenica Developmental Test of Visual-Motor Integration, também conhecido como Beery VMI. Este instrumento foi proposto para ajudar a identificar indivíduos com dificuldades nessas habilidades, servindo também para avaliar a efetividade de programas de intervenção educacional. Pode ser validamente administrado tanto em grupo de triagem quanto em propostas de avaliação individual de crianças e adultos. Apresenta dois testes suplementares padronizados, o teste de percepção visual e o de coordenação motora que são providos como um meio estatístico de avaliar a contribuição perceptual e motora para a performance no Beery VMI (BEERY; BEERY, 2010).

A aplicação desse teste, neste presente estudo, teve a finalidade de identificação da dificuldade de percepção visual, coordenação motora e integração visuo-motora dos usuários do serviço de terapia ocupacional e avaliação dos resultados da intervenção.

\section{Procedimentos}

Os participantes foram previamente selecionados tendo como base o motivo de encaminhamento para o serviço de terapia ocupacional caracterizado por queixa de desempenho nas atividades escolares. Identificados os pacientes, e tomados os cuidados com relação às providências éticas atendendo à Resolução 196/961 , os participantes foram submetidos à aplicação do Beery VMI e seus testes suplementares durante a realização do atendimento de terapia ocupacional, com a finalidade de verificar a existência de déficits nas habilidades de integração visuo-motora, percepção visual e coordenação motora, e assim identificar possíveis demandas de intervenção.

Em virtude dos resultados obtidos com os testes aplicados, os quais apontaram para déficits em todas as funções investigadas, foi verificada a necessidade de aplicação de um programa de intervenção terapêutico ocupacional visando à melhora de tais funções. Para tanto, foi utilizado o Programa de Treinamento da Percepção Visual, Coordenação Motora e Integração Visuo-motora 
Efeito da intervenção terapêutica ocupacional junto a crianças com déficts de percepção visual, cordenação motora e integração visuo-motora

(ZAFANI; ARAÚJO, 2010) destinado para crianças a partir de quatro anos de idade com déficits em uma ou mais dessas áreas.

Esse Programa de Treinamento é composto por três partes. A primeira parte apresenta cinco grupos de (a) atividades com foco na ação de traçar seguindo os pontilhados, b) na ação de traçar linhas respeitando as margens do desenho, c) na ação de completar os diferentes tracejados iniciados em cada linha e completar as figuras d) na ação de unir pontos para reproduzir um traçado e copiar figuras a partir de um exemplo, e e) na ação de identificar figuras iguais. A segunda apresenta uma análise do conjunto de Atividades de cada grupo e a terceira apresenta um Guia de Aplicação do Programa de Treinamento, bem como uma lista de sugestões de jogos opcionais.

A aplicação do programa de intervenção ocorreu num período de quatro meses, sendo uma sessão semanal de 50 minutos, totalizando aproximadamente 14 horas de intervenção.

Na intervenção realizada junto aos participantes da pesquisa, houve uma seleção das atividades de cada grupo e dos jogos opcionais, conforme prevê o Guia de Aplicação do Programa de Treinamento, a fim de o planejamento de cada sessão de atendimento, atender às características específicas de cada participante.

Durante a intervenção, e mediante o comportamento observado e as necessidades individuais de cada participante, considerou-se necessária a repetição de algumas das atividades, visando reforçar a aprendizagem, procedimento este também previsto no Guia de Aplicação do Programa de Treinamento.

Após quatro meses, finalizada a programação de intervenção, os testes foram reaplicados com o intuito de verificar se houve melhora nas funções estimuladas.

Devido ao tremor de ação apresentado por P1 em função do quadro de Paralisia Cerebral Atáxica foi utilizado, durante as aplicações do Beery VMI e do programa de treinamento, pulseira e lápis com adição de peso para diminuição do tremor, conforme sugerido por Pereira, Araújo e Braccialli (2010).

\section{Resultados e discussão}

A tabela 1 contempla os resultados obtidos no teste de integração visuo-motora e em seus testes complementares de percepção visual e coordenação motora antes da aplicação do Programa de Treinamento e a idade compatível com a pontuação, tendo como parâmetro os dados normativos para crianças americanas. 
Na tabela 1, a pontuação obtida refere-se ao total de pontos obtidos em cada um dos testes e a idade compatível, refere-se à relação estabelecida entre a pontuação obtida e idade cronológica compatível a essa pontuação. $\mathrm{Na}$ tabela, os anos estão identificados através da letra a e os meses através da letra $\mathrm{m}$.

Tabela 1. Pontuação obtida pelos participantes nos Testes de Integração VisuoMotora, Percepção Visual e Coordenação Motora antes da aplicação do Programa de Treinamento e a idade compatível com o desempenho

\begin{tabular}{|c|c|c|c|c|c|c|}
\hline \multirow{2}{*}{$\begin{array}{l}\text { PARTICI- } \\
\text { PANTE }\end{array}$} & \multicolumn{2}{|c|}{$\begin{array}{l}\text { INTEGRAÇÃO } \\
\text { VISUO-MOTORA }\end{array}$} & \multicolumn{2}{|c|}{$\begin{array}{l}\text { PERCEPÇÃO } \\
\text { VISUAL }\end{array}$} & \multicolumn{2}{|c|}{$\begin{array}{l}\text { COORDENAÇÃO } \\
\text { MOTORA }\end{array}$} \\
\hline & $\begin{array}{l}\text { Pontuação } \\
\text { obtida }\end{array}$ & $\begin{array}{l}\text { Idade } \\
\text { compatível }\end{array}$ & $\begin{array}{l}\text { Pontuação } \\
\text { obtida }\end{array}$ & $\begin{array}{l}\text { Idade } \\
\text { compatível }\end{array}$ & $\begin{array}{l}\text { Pontuação } \\
\text { obtida }\end{array}$ & $\begin{array}{l}\text { Idade } \\
\text { compatível }\end{array}$ \\
\hline P1 & 14 & $6 a 2 m$ & 7 & $3 a 0 m$ & 13 & $5 a 2 m$ \\
\hline P2 & 7 & $3 a$ 10m & 9 & $3 a 9 m$ & 11 & $4 a 6 m$ \\
\hline
\end{tabular}

Os resultados obtidos por P1 indicaram que todas as funções investigadas encontravam-se deficitárias, ou seja, seu desempenho não estava compatível com a sua idade cronológica, principalmente em relação à percepção visual. Não se pode deixar de considerar, a influência do quadro motor de P1 no seu desempenho, principalmente nos testes da Coordenação Motora e Integração visuo-motora. Segundo Pereira (1998) a criança com Paralisia Cerebral Atáxica apresenta, invariavelmente alteração na coordenação motora que compromete o desempenho em atividades, como a escrita, tornando-a incoordenada e sem ritmo, o que consequentemente ocasiona dificuldades para a realização de atividade como as propostas neste estudo (traçar seguindo os pontilhados, traçar linhas respeitando as margens do desenho, completar figuras). Portanto, a constatação de defasagem de desenvolvimento de P1, sobretudo quanto às habilidades relacionadas à percepção visual, sinalizam a importância de uma programação de intervenção com foco nesse componente de execução.

Os resultados de $\mathrm{P} 2$ indicaram também déficits em todas as funções investigadas, ou seja, seu desempenho não estava compatível com a sua idade cronológica. Segundo Falkert, Dittmann e Seelbach-Göbel (2005) diversos estudos têm mostrado que pacientes que apresentam a Síndrome de Russel-Silver têm desenvolvimento cognitivo normal, no entanto, é identificada uma frequência maior de problemas de aprendizagem quando comparados a crianças normais. O estudo clínico e genético realizado por Prince e colaboradores (1999) mostrou que 14 de 50 pacientes com achados clínicos que vão ao encontro com a definição desta síndrome apresentavam necessidades educacionais especiais. Portanto, os resultados obtidos estão em concordância com a descrição da síndrome e reforçam a necessidade de uma programação dirigida para o exercício e treinamento dessas habilidades. 
Os resultados dos testes aplicados sugerem déficits que provavelmente poderão influenciar a aprendizagem e consequentemente o desempenho escolar dos participantes.

Diante destes resultados a intervenção terapêutica ocupacional se insere tendo como foco de ação a melhora de tais funções, visando dessa forma, um desempenho e uma participação de forma mais efetiva em sala de aula.

Os resultados obtidos reforçam a importância da intervenção destinada à melhora destas funções consideradas fundamentais para o desenvolvimento da criança e para o seu desempenho escolar.

Após a análise dos resultados dos testes, deu-se início à aplicação do Programa de Treinamento.

Finalizado o período previsto de intervenção, os testes foram reaplicados.

A tabela 2 contempla os resultados obtidos no teste de integração visuo-motora e em seus testes complementares de percepção visual e coordenação motora após a aplicação do Programa de Treinamento.

Tabela 2. Pontuação obtida pelos participantes nos Testes de Integração VisuoMotora, Percepção Visual e Coordenação Motora após aplicação do Programa de Treinamento e a idade compatível com o desempenho

\begin{tabular}{|l|l|l|l|l|l|l|}
\hline \multirow{2}{*}{$\begin{array}{l}\text { PARTICI- } \\
\text { PANTE }\end{array}$} & \multicolumn{2}{l|l|l|l|}{$\begin{array}{l}\text { INTEGRAÇÃO } \\
\text { VISUO-MOTORA }\end{array}$} & \multicolumn{2}{l|}{$\begin{array}{l}\text { PERCEPÇÃO } \\
\text { VISUAL }\end{array}$} & \multicolumn{2}{l|}{$\begin{array}{l}\text { COORDENAÇÃO } \\
\text { MOTORA }\end{array}$} \\
\cline { 2 - 7 } & $\begin{array}{l}\text { Pontuação } \\
\text { obtida }\end{array}$ & $\begin{array}{l}\text { Idade } \\
\text { compativel }\end{array}$ & $\begin{array}{l}\text { Pontuação } \\
\text { obtida }\end{array}$ & $\begin{array}{l}\text { Idade } \\
\text { compatível }\end{array}$ & $\begin{array}{l}\text { Pontuação } \\
\text { obtida }\end{array}$ & $\begin{array}{l}\text { Idade } \\
\text { compativel }\end{array}$ \\
\hline P1 & 17 & $7 a 6 m$ & 19 & $8 a 3 m$ & 14 & $5 a ~ 7 m$ \\
\hline P2 & 9 & $4 a 6 m$ & 13 & $5 a 1 m$ & 13 & $5 a 2 m$ \\
\hline
\end{tabular}

Ao analisarmos a Tabela 2 podemos observar que houve melhora no desempenho de ambos os participantes em todas as habilidades após a aplicação do Programa de Treinamento da Percepção Visual, Coordenação Motora e Integração Visuo-Motora (ZAFANI; ARAÚJO, 2010).

Resultados de melhora nessas habilidades após a aplicação de um programa de treinamento também foram observados no estudo de Sanghavi e Kelkar (2005) que teve como um de seus objetivos observar a efetividade de um programa de intervenção terapêutico ocupacional de treinamento da Integração 
Visuo-motora junto às crianças com distúrbios de aprendizagem. Nesse estudo, além das crianças sem problemas de aprendizagem, 32 crianças com distúrbio de aprendizagem foram avaliadas por meio da aplicação do Beery VMI. Estas crianças foram divididas em dois grupos: grupos B e C. O grupo B recebeu atendimento individualizado de Terapia Ocupacional, baseado no treinamento da percepção visual e da coordenação motora uma vez por semana, durante 12 semanas. $\mathrm{O}$ grupo $\mathrm{C}$ recebeu orientações sobre atividades que poderiam ajudar na melhora da habilidade de integração visuo-motora sem treinamento formal. Ao final das 12 semanas ambos os grupos foram reavaliados. Os resultados da reavaliação mostraram que entre a primeira e a segunda aplicação do Beery VMI houve melhora no desempenho de ambos os grupos, no entanto, a análise estatística mostrou que houve diferença significativa entre a melhora dos dois grupos.

Outro estudo, realizado em Mumbai, China, sobre a aplicação de programa de treinamento foi conduzido por Desai e Rege (2005). Um dos objetivos desse estudo foi estudar a efetividade da intervenção terapêutica ocupacional para o treinamento de habilidade de escrita de crianças com paralisia cerebral. Para tanto, os autores utilizaram o Modified Scale of Children's Readiness In Printing (SCRIPT) e o Developmental Test of Visual-Motor Integration (Beery VMI). Os sujeitos do estudo foram expostos ao protocolo de tratamento durante dois meses recebendo duas a três sessões por semana, com duração de 35 a 45 minutos. Entre as atividades que contemplavam o treinamento, destacaramse: atividades de juntar os pontos, completar figuras e formas, fazer linhas curvas, entre outras. O estudo mostrou que os pais observaram melhora em comportamentos da criança necessários para a escrita, tais como atenção, concentração, preensão do lápis e vontade de sentar-se para as tarefas acadêmicas após a intervenção terapêutica ocupacional.

Um outro estudo que traz resultados semelhantes é o de Dias e Chaves (2001) que desenvolveu e implementou um programa de treinamento da percepção visual para alunos com dificuldades de aprendizagem (DA) e problemas de percepção visual associados. Participaram desse estudo 38 alunos com DA, com idade entre 6 e 9 anos. Nesse estudo os alunos também foram divididos em dois grupos: grupo experimental e grupo controle. O grupo experimental participou do programa durante um mês e seus integrantes receberam oito sessões individuais. Esse programa foi constituído por oito módulos de exercícios agrupados em oito categorias de capacidades de percepção visual e visuo-motoras. O grupo controle não participou do programa. Para avaliar a percepção visual antes e após o programa foi utilizado o Developmental Test of Visual Perception (DTVP-2). Os resultados das avaliações mostraram que os alunos do grupo intervenção melhoraram seu desempenho relativo à percepção visual e apresentaram resultados significantemente superiores ao grupo controle. Segundo os autores, o treino sistemático permitiu melhorar as competências perceptivas dos alunos com dificuldades de aprendizagem e este tipo de programa pode beneficiar tanto os alunos com DA como os alunos em geral que apresentarem problemas de percepção visual. 


\section{Conclusão}

Os resultados do estudo apontaram para a melhora de todas as funções consideradas deficitárias após intervenção terapêutica ocupacional, sinalizando a importância do treinamento de desempenho.

O estudo permitiu também, a reflexão sobre as contribuições da terapia ocupacional no contexto escolar junto a crianças com déficits na percepção visual, na coordenação motora e na integração visuo-motora, já que esses são componentes de desempenho considerados fundamentais para a execução das atividades escolares, estando estreitamente relacionados com as habilidades de escrita, leitura, matemática, entre outras.

Levando em conta que a intervenção seguiu um programa específico (ZAFANI; ARAÚJO, 2010), há indícios de resultados eficazes desse treinamento, recomendando-se a avaliação da eficiência desse programa em futuros estudos.

\section{Referências}

DESAI, A. S.; REGE, P. V. Correlation between Developmental Test of Visual Motor Integration [VMI] and handwriting in cerebral palsy children. Indian Journal Occupational Therapy, Malad, v. 37, n. 2, 2005.

DIAS, M. M. C. B. S.; CHAVES, J. H. S. S. O programa de treino da percepção visual para alunos com dificuldades de aprendizagem do 10 ciclo do ensino básico. In: II Conferência Internacional Challenges'2001/Desafios 2001, Anais da II Conferência Internacional Challenges'2001/Desafios 2001, p. 707721.

FALKERT, A.; DITTMANN, K.; SEELBACH-GÖBEL, B. Silver-Russell Syndrome as a cause for early intrauterine growth restriction. Prenatal Diagnosis, v. 25, n. 6, p. 497-501, 2005.

LAW, M. Participations in the occupations of everdaylife. American Journal Occupational Therapy, Bethesda, v. 56, n.6, p. 640-649, 2002.

MARTINS, L. A. R. Por uma escola aberta às necessidades dos alunos. Temas sobre desenvolvimento, São Paulo, v. 10, n. 55, p. 28-43, 2001.

MAGALHÃES, L. C. et all. Problemas de coordenação motora em crianças de 4 a 8 anos: levantamento baseado no relato de professores. Revista de Terapia Ocupacional da Universidade de São Paulo, São Paulo, v. 20, n. 1, p. 20-8, 2009.

MENDES, E. G. Perspectivas para a construção da escola inclusiva no Brasil. In: PALHARES, M. S.; MARINS, S. C. F. (Org.) Escola Inclusiva. São Carlos: Ed UFSCar, 2002. 
Rita de CássiaTibério Araújo - Mariana Dutra Zafani

Débora Morais Pereira

NOGUEIRA, L. A.; CARVALHO, L. A.; PESSANHA, F. C. L. A psicomotricidade na prevenção das dificuldades no processo de alfabetização e letramento. Pespectivas Online, Campos Goytacazes, v. 1, n. 2, p. 9-28, 2007.

PEREIRA, D. B. Terapia ocupacional em paralisia cerebral atetóide e atáxica. In: SOUZA, A. M. C.; FERRARETTO, I. (Org.). Paralisia cerebral: aspectos práticos. 2. ed. São Paulo: Memnon, 1998.

PEREIRA, D. M.; ARAÚJO, R. C. T.; BRACCIALLI, L. M. P. Adaptações funcionais de atividades para a participação escolar de crianças com disfunções neuromotoras. Revista de Educação Especial, Santa Maria, v. 23, n. 38, p. 423-42, 2010.

PRICE, S. M. et all. The spectrum of Silver-Russell syndrome: a clinical and molecular genetic study and new diagnostic criteria. Journal of Medical Genetics, p. 837-842, 1999.

ROCHA, E. F.; LUIZ, A.; ZULIAN, M. A. R. Reflexões sobre as possíveis contribuições da Terapia Ocupacional nos processos de inclusão escolar. Revista de Terapia Ocupacional da Universidade de São Paulo, São Paulo, v.14, n. 2, p.72-78, 2003.

ROSA NETO, F. Manual de avaliação motora. Porto Alegre: Artmed Editora, 2002.

SANGHAVI, R.; KELKAR, R. Visual-motor integration and learning disabled children. Indian Journal Occupational Therapy, Malad, v. 37, n. 2, p. 33-38, 2005.

WEIL, M. J.; AMUNDSON, S. J. Relaltionship between visuomotor and handwriting skills of children in kindergarten. American Journal Occupational Therapy, Bethesda, v. 48, n. 11, p. 982-988, 1994.

ZAFANI, M. D.; ARAÚJO, R. C. T. Programa de treinamento da percepção visual, coordenação motora e integração visuo-motora: descrição do processo de elaboração. In: IV Congresso Brasileiro de Educação Especial e IV Encontro da Associação de Pesquisadores em Educação Especial. Universidade Federal de São Carlos - UFSCAR, nov. 2010.

\section{Notas}

${ }^{1} \mathrm{O}$ trabalho foi aprovado pelo Comitê de Ética em Pesquisa da Faculdade de Filosofia e Ciências, UNESP - Marília, e sob o parecer n. 1959/2009.

\section{Correspondência}

Débora Morais Pereira - Rua Enseada, 379 - Jardim Satélite, São José dos Campos, CEP 12230-630, São Paulo, Brasil.

E-mail: debora_terapi@yahoo.com.br 